\title{
In Vivo Data Acquisition Instrument for Solid Organ Mechanical Property Measurement
}

\author{
Mark P. Ottensmeyer ${ }^{1}$ and J. Kenneth Salisbury, Jr. ${ }^{2}$ \\ 1 Simulation Group, CIMIT, HGX-2, Massachusetts General Hospital \\ 55 Fruit St., Boston, MA 02114, USA, \\ mpo@alum.mit.edu \\ 2 Intuitive Surgical, Inc., 1340 W. Middlefield Rd., Mountain View, CA, 94043, USA
}

\begin{abstract}
Surgical simulation systems need not only models that capture the behaviors of living tissue, but also parameters measured from real tissues to make such models meaningful. A portable system called the TeMPeST 1-D (Tissue Material Property Sampling Tool) that can acquire force-displacement responses in vivo has been developed. By fitting these data to the form of a chosen model, the tissue parameters can be obtained.

The data acquisition tool is suitable for minimally invasive or open surgical use. It measures normal indentation force-displacement response over a frequency range from DC to approximately $100 \mathrm{~Hz}$. This permits the investigation of the visco-elastic properties of living solid organ tissue. It can exert forces up to $300 \mathrm{mN}$, and has a range of motion of $\pm 500 \mu \mathrm{m}$. The TeMPeST 1-D was used to measure the frequency-dependent stiffness of porcine liver in vivo in a proof-of-concept demonstration, and is being used in a more comprehensive series of tests. Based on simple tissue models, preliminary estimates for tissue stiffness are presented and the frequency-dependent and non-linear characteristics are discussed.
\end{abstract}

\section{Introduction}

Our work has focused on providing support for the development of a simulator for laparoscopic surgery. Some of the structures of interest include solid organs such as liver, spleen and kidney. This paper will describe a surgical instrument that permits the measurement of the mechanical response of tissue in vivo. By substituting the response data into a suitable tissue model, the parameters characterizing the tissue can be determined.

Generally speaking, surgical simulations that generate accurate force feedback and deformations depend on knowledge of the mechanical properties of biological tissues. While many groups are developing such simulators ([8], [5], [11]), most must rely on estimated values or measurements typically made in vitro. Various reviews of tissue properties include [3], [21] and [4], but these and most other sources of data, use cadaver or animal tissues. The properties of non-living tissues are known to be significantly different from those of living organisms, due to temperature and strain state differences, and the absence of 
blood perfusion, among other factors ([21], [4]). To provide the necessary data, tools must be developed to measure properties in the living state.

Some of the groups pursuing these data are using MRI, ultrasound and other imaging methods that scan broad regions of the tissue to measure properties such as elastic (Young's) or shear moduli ([18, [20], [19]). By making a static or oscillatory deformations and simultaneously scanning, the strain field in the tissue and tissue moduli can be calculated. However, for the static techniques, only relative values are available and no technique covers the range of frequencies relevant to haptic feedback (i.e. DC to $100 \mathrm{~s}$ of $\mathrm{Hz}$ ). The relationship between elasticity and sonic velocity in a material has been investigated with limited success [9].

Other groups are developing tools to measure organ properties by imposing deformations in a variety of ways. The Dundee Single Point Compliance Probe (DSPCP), a hand-held tool 2], records indentation depth and force while the user presses a rigid indenter against tissue. Frequency dependence cannot be determined, but the DSPCP has a range of motion sufficient to investigate nonlinearity in stiffness. Stiffness could also be measured with a piezoelectric tube device [16]. Anisotropy in stiffness is related to the variation in the cantilevered tube's resonant frequency with direction of vibration tangential to the tissue surface. A number of groups have modified laparoscopy tools [17], [15] to measure the force-displacement characteristics of tissue, but typically with the intention of augmenting the sensations perceived by the surgeon rather than determining material properties. Recent results on porcine liver and spleen are beginning to be presented [1, as well as data and models for porcine brain [10].

Due to the limited data and lack of standard instruments, we developed the TeMPeST 1-D (Tissue Material Property Sampling Tool) to precisely deform tissues and record the force-displacement response ([12], [13], [14]). This device will be described in the next section.

\section{TeMPeST 1-D: Tissue Material Property Sampling Tool}

The TeMPeST 1-D is a minimally invasive tool that passes through standard $12 \mathrm{~mm}$ cannulas and can be fixed to the operating table with a modified laparoscope holder. It is designed to have a range of motion of $\pm 500 \mu \mathrm{m}$ and an open-loop bandwidth of approximately $100 \mathrm{H} 21$. The small range of motion was intended to cover the linear regime of tissue response, thus enabling determination of the linear elastic modulus, and also to investigate whether the limits of linearity lay within this range.

The TeMPeST 1-D makes use of a voice-coil linear actuator to drive a right cylindrical indenter. The maximum force that can be exerted is $0.3 \mathrm{~N}$, with a resolution of approximately $70 \mu \mathrm{N}$. The device can impose sinusoidal, chirn 2 ,

\footnotetext{
${ }^{1}$ varies slightly depending on stiffness of material being tested

2 sinusoidal signal with time-varying frequency
} 

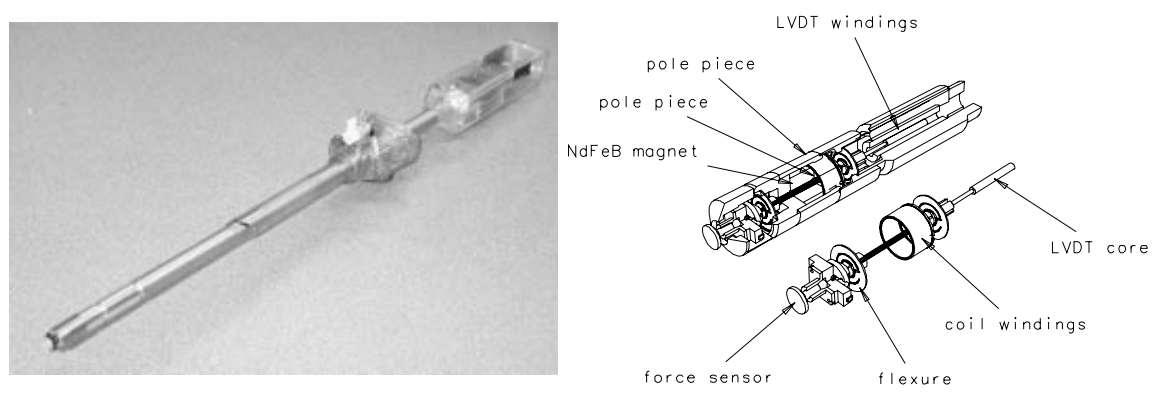

Fig. 1. TeMPeST 1-D in 12mm cannula (left) and details of indenter, actuator, force sensor and LVDT (right) showing cut-away sensor/actuator package and details of moving core.

step, and other force profiles on the tissue, while independently recording the force exerted with a force sensor at the tip, and the deformations imposed with an LVDT position sensor (see Figure回). Force and position measurement resolutions are $\pm 70 \mu \mathrm{N}$ and $\pm 0.2 \mu \mathrm{m} 3$

As an ideal case, tissue can be modeled as a linear elastic, isotropic, homogeneous and incompressible ([18, [20]) medium. In this instance, only Young's modulus is required to describe the material. If force and deformation are known, and if the tissue geometry can be approximated by a semi-infinite body undergoing normal indentation by a right circular punch, the parameters are related by equation (11) 6], as illustrated in Figure 2

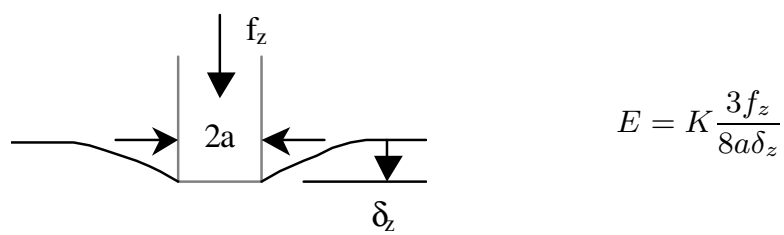

Fig. 2. Indentation of semi-infinite elastic body with rigid, right circular punch.

$\delta_{z}$ and $f_{z}$ are the displacement and force normal to the surface, $a$ is the cylindrical indenter radius, and $E$ is Young's modulus. For a semi-infinite body, $K$ is unity [7], but if the elastic material is a layer of thickness $h$ bonded to a flat, rigid surface, $K$ increases with increasing $a / h$ and $\delta_{z} / h$.

Since living tissue is known to have viscous and other dissipative mechanisms, it may exhibit a frequency-dependent sinusoidal response. In the case of normal indentation, the static expression of equation (1) can be extended to the dynamic case through the correspondence principle [4], so that $E(\omega)$ is determined from

\footnotetext{
${ }^{3}$ A 12-bit motion controller card was used for control and data input.
} 

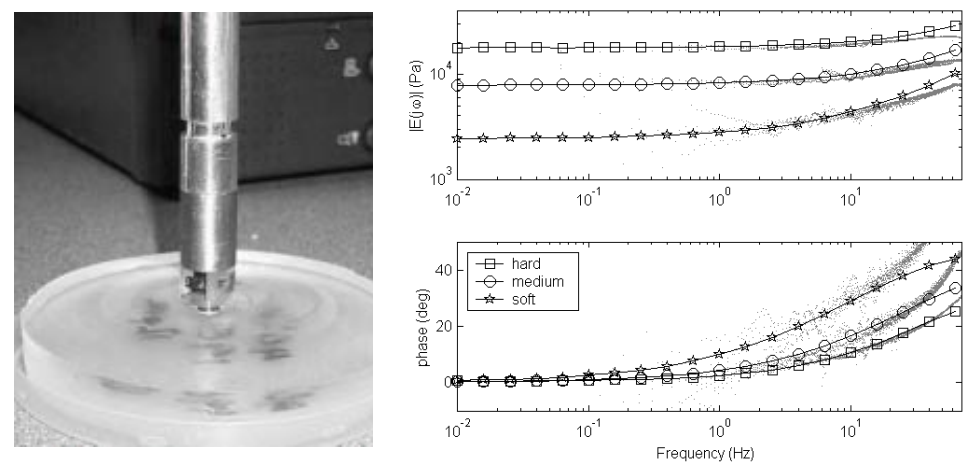

Fig. 3. TeMPeST 1-D testing silicone gel sample (left). Comparison of results from TeMPeST 1-D (dots) and parallel plate rheometer (symbols). Note close match between two data sets over wide frequency range (right).

$\delta_{z}(\omega)$ and $f_{z}(\omega)$. These results can provide data on damping coefficients and time constants of the material.

A preliminary survey of inhomogeneity could be made by taking data from numerous locations on the same organ, but anisotropy cannot be measured with the TeMPeST 1-D. However, until simulation systems become sufficiently powerful, providing $E(\omega)$ will be a significant first step, especially since these data will be based on living tissue.

\subsection{TeMPeST 1-D Characterization and Validation}

Before using the instrument in a surgical setting, the sensors and actuators were characterized, and comparisons were made between measurements taken with the TeMPeST 1-D and either known quantities or other independent tests. The position sensor and signal processing system were found to have a flat frequency response to at least $100 \mathrm{~Hz}$, while the force sensor and amplifier behave like a first order filter with a break frequency of approximately $380 \mathrm{~Hz}$. This behavior reflects the small output signal of the force sensor and the gain-bandwidth product limits of the instrumentation amplifier. The break frequency can be increased with better amplifiers, but the inverse of the force sensor transfer function can be applied to frequency-dependent measurements to compensate for the filtering effect. Finally, the actuator has both a flat frequency response and a constant current/force proportionality over its range of motion.

Tests were performed on lumped elements including a series of mechanical springs and standard laboratory masses. The measured spring frequency responses were flat, with magnitudes agreeing with static load cell tests. Impedance measurements on five and ten gram masses showed slopes of $-40 \mathrm{~dB} /$ decade and calculated masses within $10 \%$ of the correct values.

Figure 3 shows a comparison between swept-sine wave-based measurements made with a parallel plate rheometer on three silicone gel samples, each with a 


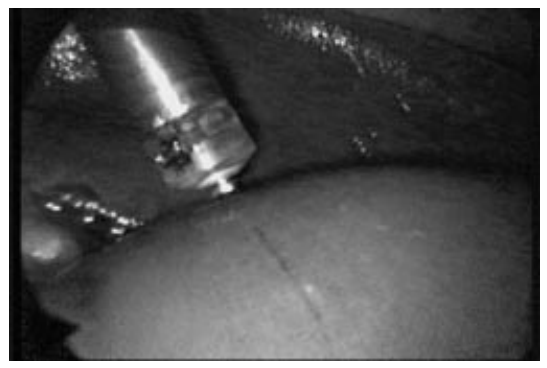

Fig. 4. Typical laparoscope view of TeMPeST 1-D approaching porcine liver.

different stiffness, with those made with the TeMPeST 1-D using chirp signals. The rheometer applies torsional loading to a thin sample of the gel, recording torque and angular displacement, from which elastic moduli can be directly calculated. The force-displacement response of the TeMPeST 1-D was converted to elastic modulus values using equation (1). Good agreement is shown between the two methods, despite completely different deformation modes. The low frequency measurements, especially for the softest materials, are subject to numerical errors, which can be reduced by increasing the sampling duration and indenter radius 4 This shows that the TeMPeST 1-D, together with the results from solid mechanics, can be used to determine acceptable values of material properties.

\subsection{In Vivo Liver Tests}

The TeMPeST 1-D has been used under both laparoscopic and open surgical conditions to measure the elastic modulus of porcine liver in vivo 5 A typical view from the laparoscope is shown in Figure 4

The initial phase of testing included tests using fixed frequency sinusoids and chirp signals. Using the semi-infinite approximation described above (equation (1)), the Young's modulus for liver, over 0.1 to $60 \mathrm{~Hz}$ is shown in Figure 5 . The lowest measured value was approximately $2.2 \mathrm{kPa}$. Simple preliminary analysis, including a least-squares fit using the less noisy sinusoidal data shows that a plane of the form of equation (3) captures the dominant characteristics of the data.

$$
\begin{aligned}
& \log _{10} E=c_{0}+c_{f} \log _{10} f+c_{\sigma} \sigma_{\text {med }}^{*} \\
& \log _{10} E=3.31+0.161 \log _{10} f+2.152 \times 10^{-4} \sigma_{\text {med }}^{*}
\end{aligned}
$$

\footnotetext{
${ }^{4}$ to increase low frequency content and applied force.

${ }^{5}$ Approval for the animal use protocols were obtained from the Dartmouth College IACUC for tests at the Dartmouth Medical School Animal Resource Center, and the Harvard University Standing Committee on Animals for tests at the Harvard Center for Minimally Invasive Surgery, as well as by the MIT Committee on Animal Care and the USAMRMC to permit the participation of the first author in the testing.
} 

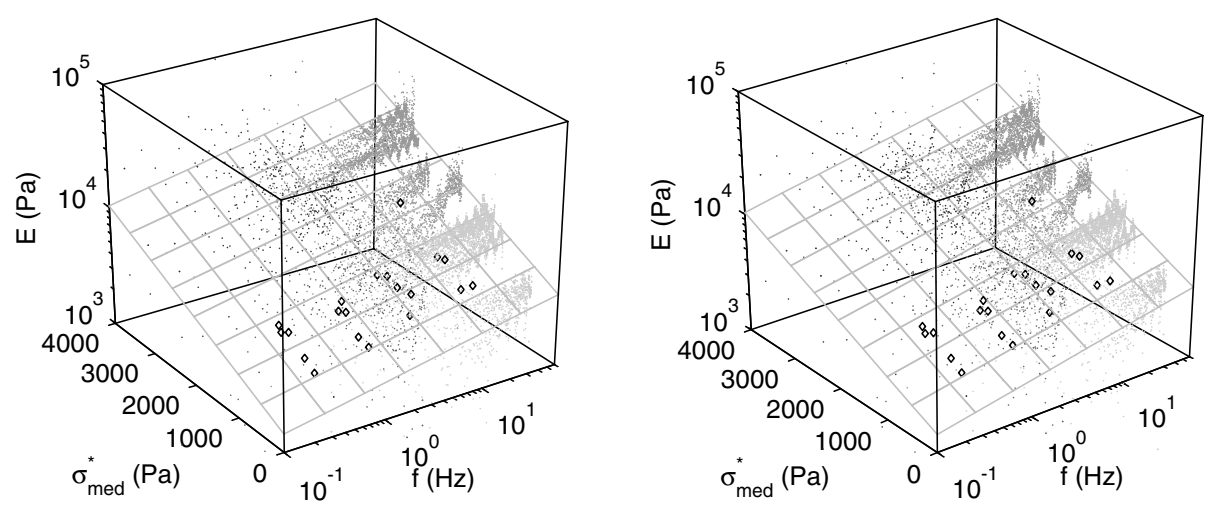

Fig. 5. Elastic modulus based on semi-infinite elastic body approximation, showing variation with both frequency and median nominal applied stress. Fixed frequency sinusoidal tests labeled by $\diamond$, chirp transform data by shaded clouds of points. Shading corresponds with median nominal stress (y-axis) value. This image can be viewed as a stereogram by crossing one's eyes.

$E$ is the elastic modulus, $f$ the frequency in Hertz, and $\sigma_{\text {med }}^{*}$ is the median nominal stress, which is the median applied force (considered over the full testing interval) divided by the cross sectional area of the indenter tip.

\section{Discussion and Conclusions}

The TeMPeST 1-D has been designed to measure mechanical properties of solid organ tissue in the living state. The instrument was tested on a series of standard materials, demonstrating that its measurements corresponded with independent tests. It has further been used to measure the elastic modulus of porcine liver in vivo. The results obtained are more complex than pure linear elastic or standard linear visco-elastic tissue models, but the measured range of elasticities falls within that expected from limited available in vitro data.

The preliminary expression used to fit the data has a number of characteristics which bear comment. First, there is an increase in modulus with frequency, likely due to viscous behavior of the tissue. While this is expected, the available data do not indicate what the minimum, steady-state value is. Additional tests at lower frequencies are required to address this issue. Second, there is a non-linear, exponential relationship between the nominal applied stress and elasticity. Because linear elasticity was expected, absolute depth of indentation was not measured, only the relative motion from the depth established by the preload was measured. In future tests, the controller and sampling sequence will be altered so that the absolute depth can be recorded. From the fit of the available data, however, and by recognizing that $E=\frac{d \sigma}{d \varepsilon}$, equation (3) can be solved for stress as a function of strain (equation (50)). 


$$
\begin{aligned}
\ln \frac{d \sigma}{d \varepsilon} & =\frac{c_{0}}{\log e}+c_{f} \ln f+c_{\sigma} \\
\Rightarrow \sigma & =\frac{-\log e}{c_{\sigma}} \ln \left(1-\frac{c_{\sigma}}{\log e} e^{\frac{c_{0}}{\log e}+c_{f} \ln f} \varepsilon\right)
\end{aligned}
$$

These results will be validated as ongoing testing continues, and will be extended with tests at higher and lower frequencies, as well as over a wider range of applied loads. Further, due to the high uncertainties produced from the chirp measurements, fixed frequency sinusoidal measurements will be made, especially at lower frequencies. In addition, other solid organ tissues will be tested, including spleen.

While highly realistic surgical simulation is still some way off, collection of better tissue property data is a crucial step along the path.

\section{Acknowledgements}

This work was supported in part by the Department of the Army, under contract number DAMD17-99-2-9001. The views and opinions expressed do not necessary reflect the position or the policy of the government, and no official endorsement should be inferred.

\section{References}

1. Brower, I., Ustin, J., Bentley, L., Sherman, A., Dhruv, N., Tendick, F.: Measuring In Vivo Animal Soft Tissue Properties for Haptic Modeling in Surgical Simulation, Medicine Meets Virtual Reality 2001, Studies in Health Technology and Informatics, 81, Newport Beach, CA (24-27 Jan 2001) 69-74

2. Carter, F.J.: University of Dundee Surgical Technology Group, 2/3/2000, last modified, 4/19/1999, http://surgtim.medschool.dundee.ac.uk/compprobe/main.html

3. Duck, F.A.: Physical Properties of Tissue, a comprehensive reference book. ISBN: 0-12-222800-6 Academy Press, Harcourt Brace Jovanovich, London (1990)

4. Fung, Y.C.: Biomechanics: Mechanical Properties of Living Tissues. ISBN: 0-38797947-6, Springer-Verlag, New York (1993)

5. Gibson, S., Samosky, J., Mor, A., Fyock, C., Grimson, E., Kanade, T., Kikinis, R., Lauer, H., McKenzie, N., Nakajima, S., Ohkami, H., Osborne, R., Sawada, A.: Simulating arthroscopic knee surgery using volumetric object representations, realtime volume rendering and haptic feedback. Proceedings of First Joint Conference, Computer Vision, Virtual Reality and Robotics in Medicine and Medical Robotics and Computer-Assisted Surgery, Grenoble, France (19-22 Mar. 1997) 369-78

6. Hayes, W.C., Keer, L.M., Herrmann, G., Mockros, L.G.: A Mathematical Analysis of Indentation Tests of Articular Cartilage, Journal of Biomechanics, 5 (1972) 541-51

7. Johnson, K.L.,: Contact Mechanics. ISBN: 05-2125576-7, Cambridge University Press, Cambridge (1985)

8. Kühnapfel, U.G., Kuhn, Ch., Hübner, M., Krumm, H.-G., Maaß, H., Neisius, B.: The Karlsruhe Endoscopic Surgery Trainer as an example for virtual reality in medical education. Minimally Invasive Therapy and Allied Technologies, 6 (1997) 122-5 
9. Maaß, H., Kühnapfel, U.G.: Noninvasive measurement of elastic properties of living tissue, Computer Assisted Radiology and Surgery (CARS '99): proceedings of the 13th international congress and exhibition (1999) 23-6

10. Miller, K., Chinzei, K., Orssengo, G., Bednarz, P.: Mechanical properties of brain tissue in-vivo: experiment and computer simulation. Journal of Biomechanics 33 (2000) 1369-76

11. O’Toole, O., Playter, R., Krummel, T., Blank, W., Cornelius, N., Roberts, W., Bell, W., Raibert, M.: Assessing skill and learning in surgeons and medical students using a force feedback surgical simulator, Proceedings of Medical Image Computing and Computer-Assisted Intervention - MICCAI'98. First International Conference, Cambridge, MA (11-13 Oct. 1998) 899-909

12. Ottensmeyer, M.P.: Minimally Invasive Instrument for In Vivo Measurement of Solid Organ Mechanical Impedance, Doctoral Thesis, Department of Mechanical Engineering, MIT (2001)

13. Ottensmeyer, M.P., Ben-Ur, E., Salisbury, J.K.: Input and Output for Surgical Simulation: Devices to Measure Tissue Properties in vivo and a Haptic Interface for Laparoscopy Simulators, Proceedings of Medicine Meets Virtual Reality 2000, Studies in Health Technology and Informatics, 70, Newport Beach, CA (27-30 Jan 2000) 236-242

14. Ottensmeyer, M.P., Salisbury, J.K.: In vivo mechanical tissue property measurement for improved simulations, Proceedings of Digitization of the Battlespace V and Battlefield Biomedical Technologies II, R. Suresh and H.H. Pien, Eds., Proc. SPIE 4037, Orlando, FL (24-28 Apr 2000) 286-293

15. Rosen, J., Hannaford, B., MacFarlane, M.P., Sinanan, M.N.: Force Controlled and Teleoperated Endoscopic Grasper for Minimally Invasive Surgery-Experimental Performance Evaluation, IEEE Transactions on Biomedical Engineering, 46(10) (1999) $1212-1221$

16. Sarvazyan, A., Schafer, M.E., Ponomarev, V.: Method and device for measuring anisotropic mechanical properties of tissue, United States Patent 5,706,815 (13 Jan 1998)

17. Scilingo, E.P., DeRossi, D., Bicchi, A., Iacconi, P.: Haptic display for replication of rheological behavior of surgical tissues: modelling, control, and experiments, Proceedings of the ASME Dynamics, Systems and Control Division, Dallas, TX (16-21 Nov 1997) 173-176

18. Skovoroda, A.R., Lubinski, M.A., Emelianov, S.Y., O’Donnell, M.: Reconstructive elasticity imaging for large deformations, IEEE Transactions on Ultrasonics Ferroelectrics \& Frequency Control, 46(3) (1999) 523-35

19. Suga, M., Matsuda T., Okamoto, J., Takizawa, 0., Oshiro, O., Minato, K., Tsutsumi, S., Nagata, I., Sakai, N., Takahashi, T.: Sensible Human Projects: Haptic Modeling and Surgical Simulation Based on Measurements of Practical Patients with MR Elastography-Measurement of Elastic Modulus, Medicine Meets Virtual Reality 2000, Studies in Health Technology and Informatics, 70, Newport Beach, CA (27-30 Jan 2000) 334-40

20. Sumi, C., Suzuki, A., Nakayama, K.: Estimation of shear modulus distribution in soft tissue from strain distribution, IEEE Transactions on Biomedical Engineering, 42(2) (1995) 193-202

21. Yamada, H.: Strength of Biological Materials. SBN: 683-09323-1, Williams \& Wilkins Company, Baltimore (1970) 